\title{
Feedback-assisted MAC protocol for real time traffic in high rate wireless personal area networks
}

\author{
Byung-Seo Kim · Sung Won Kim • Yuguang Fang • \\ Tan F. Wong
}

Published online: 17 June 2009

(C) Springer Science+Business Media, LLC 2009

\begin{abstract}
During the past decade, there has been much standardization effort for indoor or shot-range networks, as communication devices and applications for such networks populate. As a prominent example of these activities, the IEEE 802.15.3 Task Group (TG) published a standard for high-rate wireless personal area network (HR-WPAN). To support strictly timed multimedia services, the TG adopts a time-slotted channel access protocol controlled by a central device (DEV). Although the channel time allocation algorithm plays a key role in deciding the network performance, it remains unspecified in the standard. Therefore, in this paper, we propose a novel feedback-assisted channel time allocation method for HR-WPAN. After initial channel times are allocated based on packet inter-arrival time statistics, the allocation is dynamically adjusted by utilizing feedback information from each DEV. The feedback information includes the buffer status, the packet transmission delay, and the physical transmission rate. By
\end{abstract}

\footnotetext{
B.-S. Kim $(\bowtie)$

Department of Computer and Information Communication Engineering, HongIk University, JochiwonUb,

ChungCheongNamDo 339-701, Korea

e-mail: jsnbs@hongik.ac.kr

S. W. Kim

School of Electrical Engineering and Computer Science, Yeungnam University, Gyeongsangbuk-do 712-749, Korea e-mail: ksw@ieee.org

Y. Fang · T. F. Wong

Department of Electrical and Computer Engineering, University of Florida, Gainesville, FL 32611-6130, USA

Y. Fang

e-mail: fang@ece.ufl.edu

T. F. Wong

e-mail: twong@ece.ufl.edu
}

utilizing this feedback information, the central DEV can allocate sufficient channel time for transmissions of pending packets from a DEV. Moreover, the allocated channel times can be synchronized to the packet arrival times so that the overall transmission delay is reduced. To cope with time-varying wireless channels, a dynamic rate selection algorithm assisted by physical layer information is proposed in this paper. Performance evaluation is carried out through extensive simulations, from which significant performance enhancements are observed.

Keywords Wireless Personal Area Network (WPAN) . Wireless MAC $\cdot$ Link adaptation $\cdot$ Piconet

\section{Introduction}

Wireless connectivity has revolutionized consumer electronics and personal computer peripherals. High performance wireless networking solutions replace today's wired devices such as Universal Serial Bus (USB) and 1394 with greater flexibility and simpler installation. In addition, supported by emerging standards such as 802.11 and 802.15 , it is anticipated that wireless technology will eventually be used to replace the tangle of wires needed to transfer video and audio signals.

Wireless Personal Area Networks (WPANs) being studied by the IEEE 802.15 Working Group (WG) provides short range wireless connectivity among consumer electronics and communication devices. The radio range of WPAN is from 5 to $50 \mathrm{~m}$ [1]. The first standard of the IEEE 802.15 WG is IEEE 802.15.1 [2] which is a technology based on Bluetooth. The features of this technology are low power consumption, low data rate, low cost and small package size. The data rate of Bluetooth is up to $1 \mathrm{Mbps}$. 
The next generation technology of WPAN targets to consumer electronics and portable communication devices which need higher data rates. The IEEE 802.15.3 Task Group (TG) has been chartered to create a High-Rate WPAN (HR-WPAN) standard and has published a final standard [3] recently. The target applications of HRWPAN can be divided into two categories. The first application is a multi-megabyte data file transfer such as image and music files. The second application is distribution of real-time video and high-quality audio, which are strictly time-bounded applications. To support higher data rates and better Quality of Service (QoS), HR-WPAN adopts a Time Division Multiple Access (TDMA)-based Medium Access Control (MAC) protocol that will be described in Sect. 2. In HR-WPAN, a pair of nodes can communicate through peer-to-peer connectivity without contention during an allocated channel time. As the qualities of video and audio improve, the amount of data to deliver between consumer electronics also increases so that higher speed wireless connectivity is required. At this point, as the Federal Communications Commission (FCC) approved the commercial use of Ultra Wide Band (UWB) technology [4], the IEEE 802.15.3a Study Group (SG) was established to study Ultra Wide Band (UWB) technology for use in the physical (PHY) layer in HR-WPAN. Using UWB technology, the maximum achievable data rate around $500 \mathrm{Mbps}$ is suggested in [5]. Combined with the high date rate of UWB, the multimedia-oriented features of the 802.15.3 medium access controller provide the QoS provisions needed for streaming High-Definition Television (HDTV) and other multimedia applications.

Although the MAC protocol in the IEEE 802.15.3 standard is expected to play a crucial role for the formation of home networks or small office networks, significant efforts for improving the performance of the MAC protocol have not been made since the standard is recently published. Performance enhancements by informing queuestatus (Q-status) of each node to a piconet controller (PNC) are shown in [6]. In this scheme, the number of pending packets at each DEV is included in the MAC header of every packet. Thus, by overhearing every packet exchange, a PNC can allocate appropriate channel time for transmitting packets stored at a DEV in the next superframe. This scheme aims at handling Variable Bit Rate (VBR) traffics and adopts a flexible superframe size. One potential drawback is that the size of superframe may change too frequently. This may introduce some difficulties in accurate timing and positioning for strictly time-bounded applications as suggested in [7] and [8]. Furthermore, the piggybacked information can be useful only when there is a burst to transmit. Moreover, the channel time allocation algorithm for different traffic types is not considered. An algorithm proposed in [8] focuses on utilizing wasted or remaining channel times. The algorithm in [8] uses a constant superframe size. A superframe with two static channel times, one for CBR traffic and the other for realtime VBR (rt-VBR) traffic, is used. This scheme does not consider how to allocate the channel times. The authors in [9] and [10] propose a channel time allocation scheme for the specific application, MPEG 4 traffic. Since packets generated from a MPEG 4 encoder are classified into three types and are arranged in a periodic pattern, a central device can allocate channel time for transmissions of MPEG 4 packets according to the packet pattern and decoding dependency. A packet transmission method without a preamble is introduced in [11] because the physical preamble overhead remains as a dominant factor to overcome in the high transmission rate UWB technology. A rate-adaptive MAC protocol for HR-WPAN is proposed in our previous work [12]. Based on the channel quality estimated using the received packet, the receiver chooses an appropriate data rate and sends it back to the transmitter. The target applications considered in [12] are asynchronous bursty data transmission requiring an acknowledgment feedback. However, this method is not applicable for real-time services which do not require acknowledgment feedback. In [13], authors provide theoretical analysis of Delay-ACK in IEEE 802.15.3 standard.

In this paper, we propose an enhanced MAC protocol for HR-WPAN to efficiently support strictly time-bounded services and to adapt the physical transmission rate according to the time-varying channel condition. In the next section, the MAC protocol in the IEEE 802.15.3 standard is briefly described. In addition, the way to support multi-rates defined in the standard is illustrated in the same section. With the issues in the MAC protocol of the IEEE 802.15.3 standard described, the proposed MAC protocol for HR WPAN is introduced in Sect. 3. Section 4 describes the simulation environment under which the performance of the proposed protocol is evaluated. Finally, conclusions are provided in Sect. 5.

\section{IEEE 802.15.3 (high-rate WPAN)}

\subsection{MAC protocol}

In the HR-WPAN standard specifications, DEVs are communicating on a centralized and connection-oriented ad-hoc network called piconet as shown in Fig. 1. One of the participating DEVs must be designated as a piconet coordinator (PNC). The PNC provides basic timing information for the operation of the piconet and manages the quality of service (QoS) for delay sensitive applications.

The MAC layer in the IEEE 802.15.3 standard employs a time-slotted superframe structure. Figure 2 illustrates the 


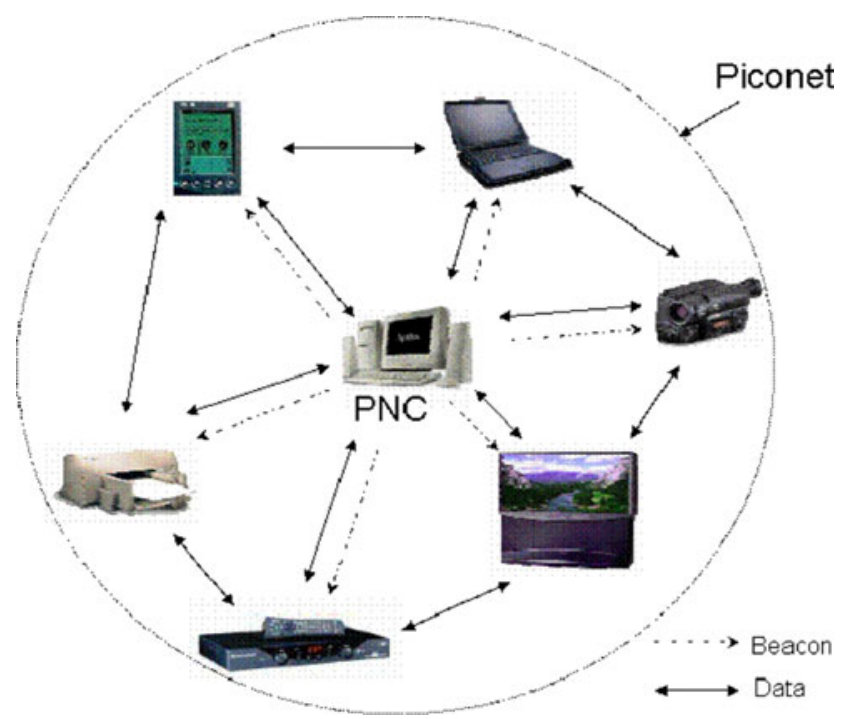

Fig. 1 A piconet in IEEE 802.15.3

superframe structure in the HR-WPAN standard. The superframe consists of three major parts: a beacon, an optional Contention Access Period (CAP) and a Channel Time Allocation Period (CTAP). The beacon packet is transmitted by the PNC at the beginning of each superframe. It allows all DEVs in a piconet to know about the specific information for controlling a piconet, such as superframe duration, channel time allocations, used frequencies and etc. The CAP is used for transmissions of short and non-QoS data packets and command/response packets. The medium access mechanism during the CAP is the Carrier Sense Multiple Access with Collision Avoidance (CSMA/CA). The remaining period in the superframe is CTAP. The CTAP is composed of Channel Time Allocation (CTA) periods and Management Channel Time Allocation (MCTA). While MCTA, like CAP, is used for sending command packets, the slotted ALOHA mechanism is used for channel access. When a DEV needs a CTA on a regular basis, it sends a channel time request (CTRq) command to the PNC during the CAP or MCTA. Thus the PNC decides the duration of the superframe, CAP, and CTAP based on the DEVs' requests. During one CTA period, one DEV can transmit several packets to one target DEV without collision. Each packet transmission may be followed by an acknowledgment (ACK) packet. A Short
InterFrame Spacing (SIFS) idle time is added for a sufficient turnaround time between two consecutive packet transmissions in a CTA. In addition to SIFS, a guard time is required to prevent collision of two adjacent CTAs. Although the scheduling algorithm for allocating CAP, MCTAs, and CTAs plays a critical role on a performance of WPAN, such algorithm is not specified in the 802.15.3 standard.

The specification for the MAC protocol defines three acknowledgment types: no-acknowledgment (No-ACK), immediate-acknowledgment (Imm-ACK) and delayedacknowledgment (Dly-ACK). An Imm-ACK is transmitted from the destination DEV when a transmitted packet is received correctly, while in the No-ACK case, no ACK is transmitted to the source DEV. A Dly-ACK is used only for directed stream data packets, i.e. isochronous connection. Figure 3 illustrates packet transmissions with No$\mathrm{ACK}$ and Imm-ACK in a CTA.

\subsection{Multi-rate support}

The IEEE 802.15.3 physical (PHY) layer is operating in the unlicensed frequency band between 2.4 and $2.4835 \mathrm{GHz}$. The symbol rate is 11 Mbaud. The raw PHY layer data rates are $11 \mathrm{Mbps}$ for uncoded QPSK modulation, and 22, 33, 44, and $55 \mathrm{Mbps}$ for trellis-coded QPSK and 16/32/64-QAM, respectively. The specification in the IEEE 802.15.3 MAC suggests two methods to obtain channel information and to select the data rate for transmission. The first method is to periodically transmit the channel status request to the target DEV. When receiving that command, the target DEV sends a channel status response back to the transmitting DEV. The channel status response includes the number of successfully received packets, the number of erroneous packets and the number of measured packets. The source DEV decides the data rate based on this information. In the second method, the channel condition is evaluated by the presence or absence of ACKs for the transmitted packets. This information is used to decide the data rate for the next packet transmissions. However, the second method is not applicable for the case using No-ACK scheme. If the DlyACK mechanism is used, all packets in a burst are transmitted with the same data rate.
Fig. 2 Superframe structure of IEEE 802.15.3

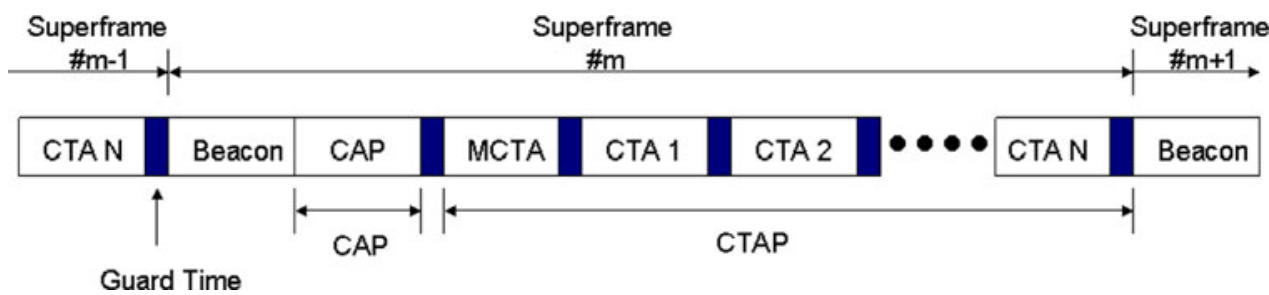


Fig. 3 Packet transmissions a with No-ACK and $\mathbf{b}$ Imm-ACK in a CTA (a)

CTA n

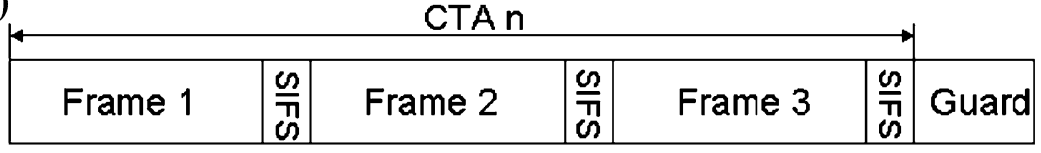

(b)

\begin{tabular}{|c|c|c|c|c|c|c|c|c|c|c|c|}
\hline Frar & $\frac{C D}{M}$ & 离 & 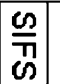 & Frame 2 & $\begin{array}{l}\frac{\omega}{\pi} \\
\boldsymbol{M}\end{array}$ & 竞 & \begin{tabular}{|l|}
$\frac{\omega}{\pi}$ \\
$\infty$
\end{tabular} & Frame 3 & 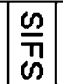 & 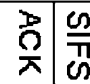 & \begin{tabular}{|l|l|}
\multirow{2}{n}{} & Guard \\
\end{tabular} \\
\hline
\end{tabular}

\section{Proposed MAC protocol}

\subsection{Motivation}

Even small delay in HR-WPAN may cause serious performance degradations since HR-WPAN is targeting on delay-constrained real time multimedia services with a bulky traffic size and high data rate, such as home theater systems with HDTV. Therefore, the channel time allocation algorithm plays an essential role to guarantee delay bound performance of real-time applications in HRWPAN. Nevertheless, such few algorithms have been proposed in the previous literatures. Furthermore, the information delivered by a CTRq command as shown in Fig. 4 is insufficient for the PNC to decide the duration and location of a CTA for the requesting DEV. The IEEE 802.15.3 TG considers the scenario that DEVs frequently join and leave a piconet as mentioned in [1]. In this scenario, many factors, such as a superframe length and the number of flows, vary in time. As a consequence, the CTA allocation algorithm is required to support the QoS requirements over these variable factors.

In wireless networks, channel conditions need to be estimated to dynamically choose the appropriate transmission data rate over the time varying wireless channel, so that the better performance can be achieved. As illustrated in Sect. 2.2, the channel condition in IEEE 802.15.3 is estimated based on the results of attempted transfers of data packets between two DEVs that are actively participating in a data transfer. However, this method cannot cope with fast channel changes and may cause incorrect channel information which leads to performance degradation. Moreover, for traffics with long packet inter-arrival time, this estimation method are futile since the transmission history for such a long time period cannot represent the current channel condition. Recently the use of Signal-to-Noise Ratio (SNR) has been suggested to estimate the channel condition. In [14], two methods, using transmission history and SNR, for the channel estimation are evaluated over a WLAN environment. The evaluation in [14] shows that the method using SNR achieves a higher performance gain than that using the result of attempted transfers of data packets. However, this formal method requires feedback information from the receiver, which is not applicable to real time applications without acknowledgments.

With these considerations, we propose an enhanced MAC protocol for time-bounded services in the next subsections.

\subsection{Proposed protocol for high-rate wireless PAN}

\subsubsection{CTA allocation algorithm}

As mentioned in the previous section, providing delaybounded services is critical to the real-time traffics and no algorithm to allocate channel times is specified in the standard. Here, we propose a channel time allocation algorithm to synchronize a CTA to the packet arrival instant. We introduce two main parameters that affect the channel time allocation process. The first one is the service period $\mathrm{IA}_{i}$ of DEV $i$. The value of $\mathrm{IA}_{i}$ is the estimated inter-
Fig. 4 Channel time request command format and channel time request block field format specified in IEEE 802.15.3 standard

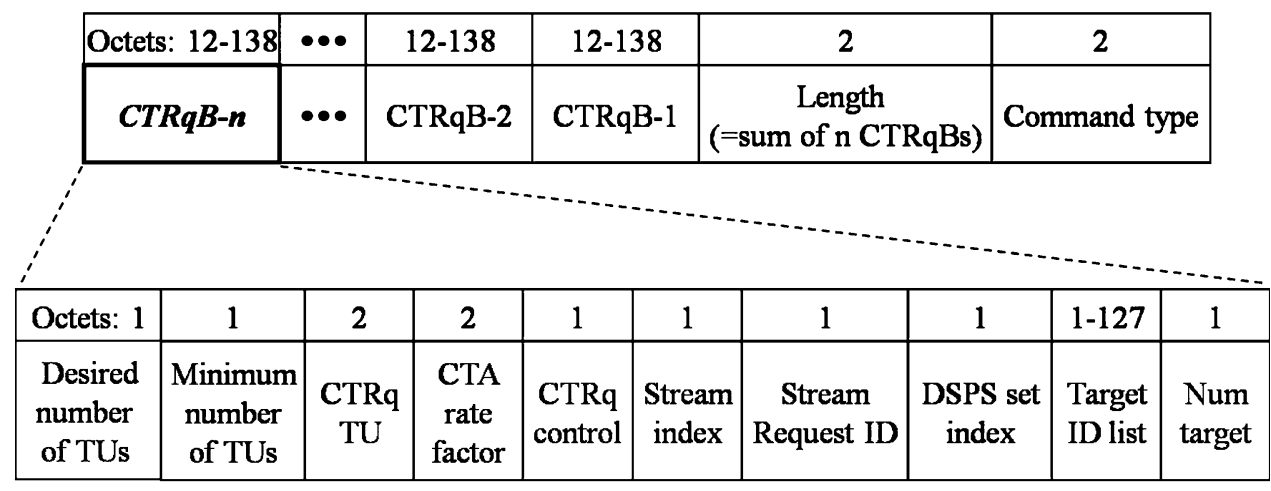


arrival time of packets at DEV $i$ with payload $P_{i}$, which is given by

$\mathrm{IA}_{i}=\left\lfloor\frac{P_{i} \times 8}{M_{i}}\right\rfloor$

where $P_{i}$ and $M_{i}$ are the payload in the MAC packet in bytes and the data arrival rate for CBR traffic (or the mean arrival rate for real-time VBR (rt-VBR) traffic) in the MAC layer at $\mathrm{DEV} i$, respectively. $\mathrm{IA}_{i}$ is calculated by $\mathrm{DEV} i$ and used to inform the $\mathrm{PNC}$ in the channel time request. In a general wireless network, the two parameters, $P_{i}$ and $M_{i}$, can be obtained from the admission control unit in the central controller during the association period as described in [15] and [16]. For this purpose, the channel time request shown in Fig. 4 is modified. The CTA rate factor field in the channel time request is changed to the Traffic arrival rate field. We define another parameter $\mathrm{Ptr}_{i}$, which is related to $\mathrm{IA}_{i}$, to allocate CTA for DEV $i$. $\operatorname{Ptr}_{i}$ is a timer initialized to be $\mathrm{IA}_{i}$ and decreased as time elapses. The moment when $\operatorname{Ptr}_{i}$ reaches zero is the time instant to allocate CTA for DEV $i$. This means that the $\mathrm{Ptr}_{i}$ indicates the remaining time for the CTA allocation for DEV $i$.

At first, the PNC gathers information about DEVs whose $\operatorname{Ptr}_{i} s$ are less than the current superframe duration because CTAs of those DEVs must be allocated in the current superframe. Then, the PNC decides the number of CTAs to be allocated in the current superframe. The PNC needs information of $\mathrm{NumCTA}_{i}, \mathrm{ST}_{i}^{j}$ and $\mathrm{DT}_{i}^{j}$ for each DEV to allocate CTAs in the superframe. NumCTA ${ }_{i}$ is the required CTAs for a DEV $i$ during a superframe period and is given by

$\operatorname{NumCTA}_{i}=\left\lfloor\frac{T_{\mathrm{SF}}-\operatorname{Ptr}_{i}}{\mathrm{IA}_{i}}\right\rfloor+1$,

where $T_{\mathrm{SF}}$ is the time duration of the superframe. $S T_{i}^{j}$ is the time instant of the beginning of CTA $j$ for DEV $i$. It is defined as

$\mathrm{ST}_{i}^{j}=\operatorname{Ptr}_{i}+(j-1) \times \mathrm{IA}_{i}, 1 \leq j \leq \mathrm{NumCTA}_{i}$.

Note that $\mathrm{ST}_{i}^{j}$ is less than $T_{\mathrm{SF}}$. $\mathrm{DT}_{i}^{j}$ is the time duration to be allocated to CTA $j$ of DEV $i$. It is calculated as

$\mathrm{DT}_{i}^{j}=\left[T_{\mathrm{OH}}+T_{\mathrm{SIFS}}+\frac{\left(L_{\text {len }}^{i}+L_{\mathrm{FCS}}\right)}{R_{i}}\right] \times Q_{i}+T_{\text {guard }}$

where $T_{\mathrm{OH}}$ is the time overhead including the preamble, PHY header, MAC header, Header Check Sequence (HCS), and guard time (In the IEEE 802.15.3 standard, the value of $T_{\mathrm{OH}}$ at $11 \mathrm{Mbps}$ is different from those at the other rates), $T_{\mathrm{SIFS}}$ is the SIFS idle time, $L_{\text {len }}^{i}$ is the length of the payload in bits for DEV $I, L_{\mathrm{FCS}}$ is the length of the frame check sequence (FCS), $R_{i}$ is the data rate in the physical layer, and $Q_{i}$ is the number of packets to be transmitted during CTA $j$ of DEV $i$. The beacon packet in a superframe has information fields for the location and duration of all
CTAs as described in the IEEE 802.15.3 standard. Thus, the proposed scheme can be implemented without any additional modification to the standard.

Now, CTAs are allocated at time $\mathrm{ST}_{i}^{j}$ with duration $\mathrm{DT}_{i}^{j}$ on a superframe. When several CTAs overlap, the CTA with lower $\mathrm{ST}_{i}^{j}$ is allocated in advance of the one with higher $\mathrm{ST}_{i}^{j}$. However, the CTAs can also be allocated based on the same specified performance requirements such as priority and throughput. In the former case, CTAs of DEV with higher priority are allocated ahead of those from another DEV with lower priority. In the latter case, CTAs of a DEV with a higher transmission data rate is allocated ahead of one with lower data rate. If there is time remaining between two consecutive CTAs, this duration becomes MCTA for transmitting command packets. However, if the remaining time is less then the threshold $T_{\mathrm{thr}}$, it is merged to previous or next CTA. Therefore, MCTA allocation is also defined. The threshold $T_{\mathrm{thr}}$ is the sum of the slot time and the time duration of a CTRq packet. This choice ensures that at least one command packet can be transmitted in the MCTA. The total duration of CTAs and MCTAs allocated in a superframe should be less than $T_{\mathrm{SF}}$. If this total duration is less than $T_{\mathrm{SF}}$, then some CTAs near the end will be removed from the allocation and will be scheduled in the next superframe.

At the final step, $\operatorname{Ptr}_{i}$ is reset to a value for the next superframe formation given by

$\operatorname{Ptr}_{i}=\mathrm{IA}_{i}-\left(T_{\mathrm{SF}}-\mathrm{ST}_{i}^{\text {last }}\right)$,

where $\mathrm{ST}_{i}^{\text {last }}$ is the time duration of the previously allocated CTA for DEV $i$. For a DEV whose CTAs are not allocated in this superframe, the corresponding $\operatorname{Ptr}_{i}$ is subtracted by $T_{S F}$. Figure 5 shows an example of the CTA allocation process for DEV 1 mentioned above. 3 CTAs are allocated for DEV 1 in the example.

\subsubsection{Feedback-assisted CTA allocation}

Employing CTA allocation algorithms based only on statistical packet inter-arrival time is not sufficient to overcome the aforementioned problem for strictly timebounded services. Since information given by a channel time request does not include the optimal time instant of a CTA, the PNC may allocate the CTA at any position within a superframe. This causes time waste from packet arrival at the MAC layer to the transmission of that packet. This wasted time is called transmission delay. Figure 6 shows an example of transmission delay caused by the lack of information about the actual packet arrival instant at the PNC. This delay increases as the packet inter-arrival time increases and may wait until the end of the flow. Furthermore, it can be longer in heavy load cases since several CTAs may overlap. Because of this problem, rt-VBR 
Fig. 5 An example of CTA allocation process for DEV 1

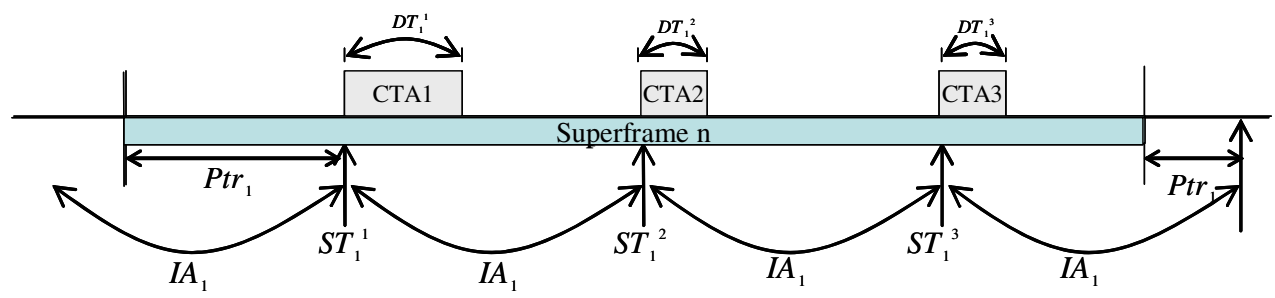

traffics whose packet inter-arrival times are variable cannot be handled satisfactorily. For rt-VBR traffic, instantaneous bit rate fluctuates widely about a mean value as shown in [17]. As a consequence, the inter-arrival time at DEV $i$ also fluctuates and is different from $\mathrm{IA}_{i}$ statistically calculated by the PNC. That means that more than one packet can be stored in the buffer at the instantaneous CTA allocation. If PNC allocates CTAs for rt-VBR traffic using the peak inter-arrival time, the utilization of channel time will be degraded.

To overcome these problems, we propose a feedbackassisted CTA allocation method. To achieve better CTA allocation, each DEV informs the PNC its current status. For this purpose, during the MCTA, a DEV sends the status information to the PNC by using the status report shown in Fig. 7. This command packet specifies three statuses of a DEV: Q-status, Delay, and Rate. Q-status indicates the number of packets in the queue. Delay is the time period from the instant of packet arrival at MAC layer to the beginning of allocated CTA. Rate is the physical transmission rate. The Report ID subfield in the status report indicates one of seven possible report types and the Report Payload subfield is the value of each reporting item. Table 1 lists the Report ID and the size of Report Payload. When the PNC receives a status report with the delay information from DEV $i$, the value of $\operatorname{Ptr}_{i}$ of DEV $i$ at the PNC is subtracted by that delay. Hence, a CTA for DEV $i$ in the next superframe will be allocated earlier than the current CTA position since $\operatorname{Ptr}_{i}$ is shortened by using the status report.

Figure 6 illustrates an example of the CTA synchronization process with packet arrivals. In the first superframe,

\begin{tabular}{|c|c|c|c|c|}
\hline Octets: 10 & 1 & $1 \sim 4$ & 2 & 4 \\
\hline MAC header & $\begin{array}{c}\text { Report } \\
\text { ID }\end{array}$ & $\begin{array}{c}\text { Report } \\
\text { Payload }\end{array}$ & Length & FCS \\
\hline
\end{tabular}

Fig. 7 Status report packet format

Table 1 List of report IDs and report payload sizes

\begin{tabular}{lll}
\hline Report Type & Report ID & Report Payload size (Octet) \\
\hline Q-Status & 0001 & 1 \\
Delay & 0010 & 2 \\
Rate & 0011 & 1 \\
Q-Status + Delay & 0100 & $3(1+2)$ \\
Q-Status + Rate & 0101 & $2(1+1)$ \\
Delay + Rate & 0111 & $3(2+1)$ \\
Q-Status + Delay + Rate & 1000 & $4(1+2+1)$ \\
\hline
\end{tabular}

DEVs $D 1$ and $D 2$ have the transmission delays, $T_{\text {delay }}^{1}$ and $T_{\text {delay }}^{2}$, respectively. The transmission delay information is sent during the MCTA of the first superframe. The PNC changes the time instant of the CTAs in the second superframe. Thus, from the second superframe on, CTAs are located at the packet arrival time instants and the transmission delay becomes zero. If the packet arrival rate is constant as CBR traffic, a single status report with delay information is enough for the PNC scheduler because a DEV with CBR traffic generates one packet in each interarrival time. However, for rt-VBR traffic, this assumption is not guaranteed as mentioned before. In order to dynamically allocate the duration of CTAs for DEVs with rt-VBR, the queue status of each DEV needs to be reported
Fig. 6 An example of CTA synchronization

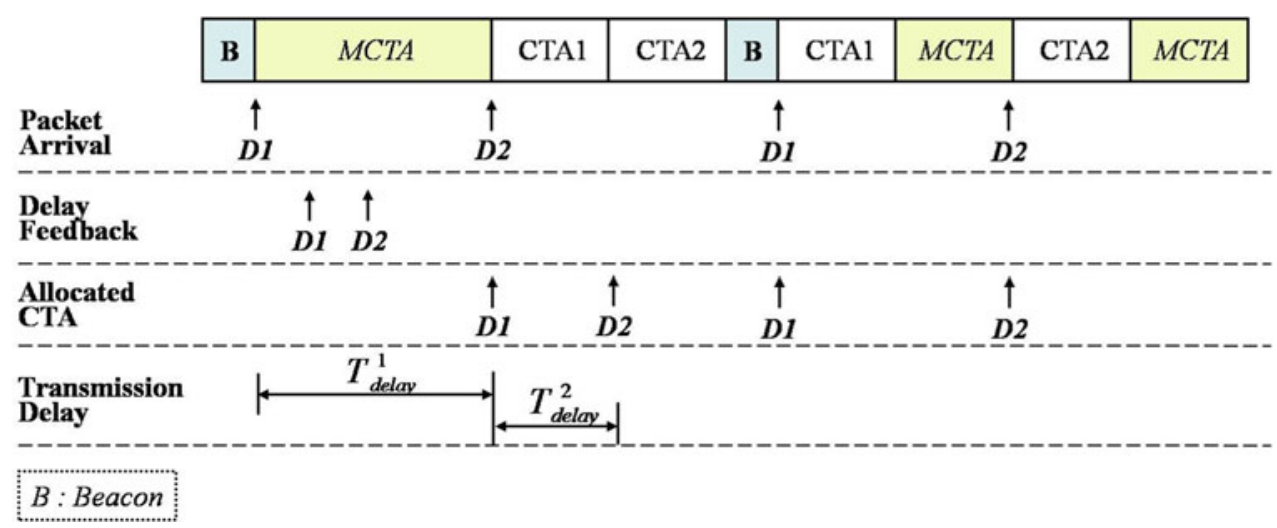


to the PNC scheduler frequently. This queue status information is also transmitted using the status report command during the MCTA. This information is used in (4) to provide the value for the parameter $Q_{i}$.

We use channel estimation from the physical layer at a receiver to choose the transmission data rate. In our previous work, a rate adaptation mechanism for best effort traffic types such as the bulk file transfer is proposed [12]. On the other hand, since we are dealing with time-bounded real-time services with No-ACK policy here, a packet to inform the data rate to the sender is needed. For this purpose, the aforementioned Status Report command is used to report the selected data rate to the PNC as well as the sender. This command is transmitted during a CAP or MCTA only when the currently used rate is not appropriate to meet certain performance criteria such as the Packet Error Rate (PER) in [12] and [18]. The channel estimation process is done by the physical layer. This feedback rate information is utilized to determine the CTA durations in the next superframe as shown in (4).

In the proposed scheme, the transmission of status report commands plays an important role in allocating CTAs in a superframe. However, the PNC may form a superframe without any MCTA due to a heavy traffic load or an insufficient superframe size. To ensure at least one status report command can be transmitted in a superframe, the PNC allocates at least one MCTA with the minimum MCTA time duration. Moreover, the last channel time in a superframe must be a MCTA, called Essential MCTA (EMCTA). This allows the latest status information of each DEV to be delivered to the PNC and reflected in the next superframe.

\section{Performance analysis}

\subsection{Networking setting}

We assume that all DEVs except the PNC are uniformly distributed in the coverage area of a piconet with diameter $20 \mathrm{~m}$. The PNC is always located at the center of the area. We do not consider any neighboring piconet in this simulation. Moreover, perfect synchronization in the physical layer is assumed and the propagation delay is not considered. The parameters used in this simulation study are shown in Table 2. The choice of these parameters is based on the IEEE 802.15.3 standards [3].

Since the proposed scheme is designed for the timebounded services, we study two real-time traffic types, CBR and rt-VBR in the simulation. The CBR traffic flow is generated at $912 \mathrm{~kb} / \mathrm{s}$. This rate is the maximum bit rate of the MPEG audio encoder in [19]. For the rt-VBR traffic model, the trace statistics of actual MPEG-4 video
Table 2 Simulation parameters

\begin{tabular}{ll}
\hline Parameter & Value \\
\hline SIFS Time & $10 \mathrm{us}$ \\
Guard Time & $50 \mathrm{us}$ \\
Slot Time & $17.3 \mathrm{us}$ \\
MAC Header & 10 octets \\
PHY Header & 2 octets \\
Preamble & $17.5 \mathrm{us}$ \\
HCS & 16 Bit \\
FCS & $32 \mathrm{bits}$ \\
Minimum MCTA & $3 \mathrm{~ms}$ \\
\hline
\end{tabular}

streams reported in [17] and [20] are used. We use a high quality video stream from "Silence of the Lambs", which has a mean bit rate of $580 \mathrm{Kbps}$ and a peak rate of 4.4 Mbps. Each DEV has either a CBR or rt-VBR traffic flow. A DEV alternates between the two states, ON and OFF, and their durations are exponentially distributed with mean values of 20.0 and $0.05 \mathrm{~s}$, respectively. A traffic flow is generated only during ON state. At the beginning of the $\mathrm{ON}$ state, a DEV selects a destination DEV and transmits a CTRq command to the PNC during a MCTA. In this simulation, CAP allocation is not considered since it is optional in the standard [3]. In addition, three measurements for performance evaluation are considered: Job Failure Ratio (JFR), the average transmission delay, and PER. The JFR is the packet dropping rate because of missing delay bound [6] and [8]. The average transmission delay is defined as the time duration from the arrival of a packet in the MAC layer to the departure of the packet or dropping of it. It is assumed that a packet arrives at the MAC layer at the instant that it is generated. All of the simulation results in the plots are obtained with a $95.7 \%$ confidence interval.

The scheme proposed in this paper, namely FeedbackAssisted WPAN (FA-WPAN), is compared with the HRWPAN scheme suggested in [6]. HR-WPAN adopts an aggressive CTA allocation algorithm. CTA durations for both CBR and rt-VBR traffic flows are evenly allocated over the superframe duration in the allocation algorithm in [6]. However, since the rt-VBR traffic may generate more packets than the CBR traffic, it is unfair to allocate the same CTA durations for both traffics. Therefore, in this simulation, the CTA duration for the rt-VBR traffic is roughly two times longer than that for the CBR traffic. HRWPAN also allocates a MCTA of $3 \mathrm{~ms}$ duration as the first CTA in every superframe. Therefore, the duration of each CTA is

$\frac{\left(T_{\mathrm{SF}}-T_{\mathrm{bec}}-T_{\mathrm{MCTA}}\right)}{\left(1 \times N_{\mathrm{vbr}}+2 \times N_{\mathrm{cbr}}\right)} \times\left\{\begin{array}{c}2 \text { for } \mathrm{rt}-\mathrm{VBR} \\ 1 \text { for CBR }\end{array}\right.$, 
where $T_{\mathrm{bec}}$ and $T_{\mathrm{MCTA}}$ are the time durations of the beacon packet and E-MCTA, respectively. $N_{\mathrm{vbr}}$ and $N_{\mathrm{cbr}}$ are the numbers of flows of rt-VBR and CBR traffics, respectively. The position of the MCTA in HR-WPAN does not affect the performance because no command packet, except the CTRq, is considered.

Each scenario is simulated for $10 \mathrm{~min}$. For the evaluation of the rate adaptation scheme, we simulate 50 different realizations with different positions of DEVs. In every realization, the channel condition for each communication link is recalculated according to the distance between any two DEVs.

\subsection{Wireless channel model}

We employ the log-distance path loss channel model in [21]. The path loss $\overline{\mathrm{PL}}$ at distance $d$ is given by

$\overline{\mathrm{PL}}(d)[d B]=\overline{\mathrm{PL}}\left(d_{0}\right)[d B]+10 n \log \left(\frac{d}{d_{0}}\right)$,

where $d_{0}$ is the close-in reference distance and $n$ is the path loss exponent. We set $n$ to 3.3 according to the SG3a alternate PHY selection criteria in [22]. To estimate $\overline{\mathrm{PL}}\left(d_{0}\right)$, we use the Friis free space equation

$P_{\mathrm{r}}\left(d_{0}\right)=\frac{P_{\mathrm{t}} G_{\mathrm{t}} G_{\mathrm{r}} \lambda^{2}}{(4 \pi)^{2} d_{0}^{2} L}$,

where $P_{\mathrm{t}}$ and $P_{\mathrm{r}}$ are the transmit and receive power, respectively, $G_{\mathrm{t}}$ and $G_{\mathrm{r}}$ are the antenna gains of the transmitter and receiver, respectively, $\lambda$ is the carrier wavelength, and $L$ is the system loss factor which is set to 1 in our simulation. The transmit power and antenna gain are set to 0 and $0 \mathrm{dBi}$, respectively, based on [22]. The received power is

$P_{\mathrm{r}}(d)[d B m]=P_{\mathrm{t}}[d B m]-\overline{P L}(d)$.

Finally, the long-term signal-to-noise ratio is

$\mathrm{SNR}_{L}[d B]=P_{\mathrm{t}}-\overline{\mathrm{PL}}(d)-N$,

where $N$ is the noise power set to $-95 \mathrm{dBm}$.

To demonstrate the functionality of the rate adaptation scheme in our proposed protocol, the received $\mathrm{SNR}_{L}$ is varied by the Ricean fading gain $\alpha$, which is generated according to the modified Clarke and Gans fading model [23]. Under this model, the SNR of the received signal is

$\operatorname{SNR}[d B]=20 \times \log _{10} \alpha+\operatorname{SNR}_{L}[d B]$.

For the data rate in the physical layer for each communication link, we assume that the system adapts the data rate by properly choosing one from a set of modulation schemes according to the channel condition. The set of modulation schemes used in our simulation studies are BPSK, QPSK, 8QAM, 16QAM, and 32QAM.
For simplicity, we ignore other common physical layer components such as error correction coding. With $11 \mathrm{MHz}$ symbol rate and the above modulation schemes, the achieved data rates are $11,22,33,44$, and $55 \mathrm{Mbps}$, respectively, which are the same data rates in the standard. Assuming that the symbol errors within a data packet are independent, the packet error rate (PER) is related to the symbol error rate (SER) by

PER $=1-(1-\mathrm{SER})^{N}$,

where $N$ is the number of symbols in the payload of an MAC packet. We set the target FER to $8 \%$ according to the IEEE 802.15.3 standard [3]. The SER equation to determine the SNR can be found in [24]. For BPSK,

$\mathrm{SER}=Q\left(\sqrt{\frac{2 E_{s}}{N_{0}}}\right)$,

and for QPSK and M-ary QAM,

$\mathrm{SER} \leq 1-\left[1-2 Q\left(\sqrt{\frac{3 E_{s}}{(M-1) N_{0}}}\right)\right]^{2}$,

where $E_{s} / N_{0}$ is the SNR per symbol and $M$ is the signal constellation size. From the SER performance curves calculated from (13) and (14), the SNR ranges for the corresponding modulation schemes that the target SER is satisfied are given as follows,

$R=\left\{\begin{array}{l}11 \text { (BPSK), } \mathrm{SNR}<\mathrm{SNR}_{22} \\ 22(\mathrm{QPSK}), \mathrm{SNR}_{22} \leq \mathrm{SNR}<\mathrm{SNR}_{33} \\ 33(8 \mathrm{QAM}), \mathrm{SNR}_{33} \leq \mathrm{SNR}<\mathrm{SNR}_{44} \\ 44(16 \mathrm{QAM}), \mathrm{SNR}_{44} \leq \mathrm{SNR}<\mathrm{SNR}_{55} \\ 55(32 \mathrm{QAM}), \mathrm{SNR}_{55} \leq \mathrm{SNR}\end{array}\right.$

where $\mathrm{SNR}_{i}$ is the SNR threshold for the data rate $i$ to meet the target SER.

\subsection{Performance evaluation}

In this section, the proposed protocol is evaluated with three superframe sizes: 25,45 , and $65 \mathrm{~ms}$. The maximum superframe size described in the IEEE 802.15.3 standard is $65,536 \mu \mathrm{s}$. The delay bound is set to the packet inter-arrival time used in [25] and [26]. That is, all packets arriving at the MAC layer must be transmitted before the next packet arrives. The inter-arrival times are varied by changing the packet size with a constant traffic bit rate for CBR (or the mean traffic bit rate for rt-VBR). The packet sizes used in this simulation are 512, 1,024, 1,286, 1,536, 1,792, and 2,048 octets defined as the preferred packet sizes in the IEEE 802.15.3 standard.

The simulation results of the JFR are shown in Fig. 8. With $25 \mathrm{~ms}$ superframe size, the JFR in FA-WPAN is 34 to 
$7 \%$ of that in HR-WPAN for the CBR traffic and is 45 to $24 \%$ of that in HR-WPAN for the rt-VBR traffic as the inter-arrival time increases. The performance differences increase with a larger superframe size. While the performance of HR-WPAN is influenced by the superframe size, the superframe size does not significantly affect the performance of FA-WPAN. Once a CTA for a DEV is allocated in a superframe in HR-WPAN, the DEV holds its transmissions for the CTA in the next superframe. Therefore, if the delay bound is shorter than the holding time, the packet will be dropped. The effect of delay bound will be described later. On the other hand, since CTAs are allocated at the packet arrival instants in FA-WPAN, more than one CTA for a DEV may be allocated in a superframe. For CBR traffic, beacon packets and E-MCTAs are more frequently generated in a shorter superframe than in a longer one. Thus they obstruct appropriate CTA allocations. This reflects that JFR of $65 \mathrm{~ms}$ superframe is slightly lower than that of $25 \mathrm{~ms}$ superframe in Fig. 8a. However, this explanation is not applicable to the case of rt-VBR traffic. While the CTA location is a critical factor for the CBR traffic, fast changes of the CTA duration according to the Q-status is a critical factor for rt-VBR traffic. However, a CTA cannot instantly be changed by the Q-status report. Although a DEV reports the number of pending packets to the PNC, CTAs allocated for a DEV are not changed during the current superframe and consequently nontransmitted packets in the current CTA are dropped. Thus, the CTA durations in a shorter superframe can be quickly adapted comparing to a longer superframe. Therefore, the JFR of $65 \mathrm{~ms}$ superframe is higher than that of $25 \mathrm{~ms}$ superframe shown in Fig. 8b.

The performance differences between HR-WPAN and FA-WPAN are more obvious when the transmission delays are evaluated as shown in Fig. 9. Theoretically, the average transmission delay for HR-WPAN is around one half of the

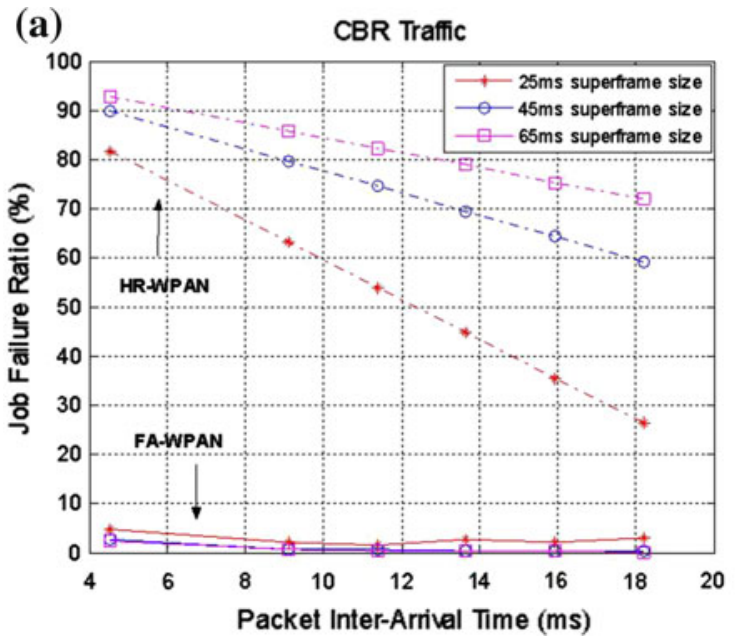

superframe size. Therefore, we observe that the gradient of the performance curve for HR-WPAN with $25 \mathrm{~ms}$ superframe reduces to about one half of the superframe size, namely, $12.5 \mathrm{~ms}$. It is also expected that the gradients of the performance curves for 45 and $65 \mathrm{~ms}$ reduce to around a half of the superframe size, which are 22.5 and $32.5 \mathrm{~ms}$, if the inter arrival time of a flow is much longer than 45 or $65 \mathrm{~ms}$. However, in our simulation, the inter-arrival times of flows are $<45$ and $65 \mathrm{~ms}$, so that it does not appear that the curves for 45 and $65 \mathrm{~ms}$ superframe sizes are saturated.

Figure 10 shows the overall network goodput performance as a function of the packet size. As mentioned before, increasing packet size results in increasing packet inter-arrival time if traffic bit rates are not changed. The results in Fig. 10 agree with the results of the JFR and transmission delays in Figs. 8 and 9.

Previous evaluations are performed using the packet inter-arrival time as the delay bound. Some applications allow longer delay constraint than the inter-arrival time as shown in [6-9]. Therefore, the performance using different delay bounds should be evaluated. For this purpose, we define a delay bound multiplier for the inter-arrival time. Here, 2,048 octets packet size is used for both traffic types. The inter-arrival times for CBR and rt-VBR traffic are 18 and $28 \mathrm{~ms}$, respectively. Figure 11 shows the JFRs of both configurations. Both the JFRs of CBR and rt-VBR in FAWPAN reaches to $0 \%$ at around 1.5 delay bound multiplier regardless of the superframe size. Although a longer delay bound generates more pending frames, the CTA duration in the $25 \mathrm{~ms}$ superframe for CBR traffic in HR-WPAN is insufficient to deal with more than two packets. Therefore, the JFRs for CBR in HR-WPAN are constant, regardless of the delay bounds. Except for CBR traffic case in HRWPAN, the JFRs in HR-WPAN reach to $0 \%$ at longer delay bound multiplier than in FA-WPAN. Considering the delay bound of $30 \mathrm{~ms}$, the commonly used maximum delay

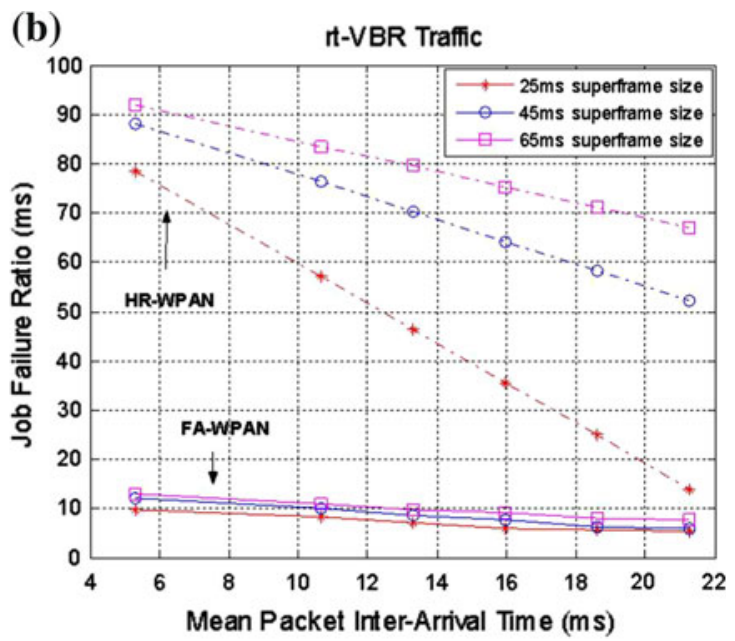

Fig. 8 Job Failure Rate as a function of the packet inter arrival time for different superframe sizes 

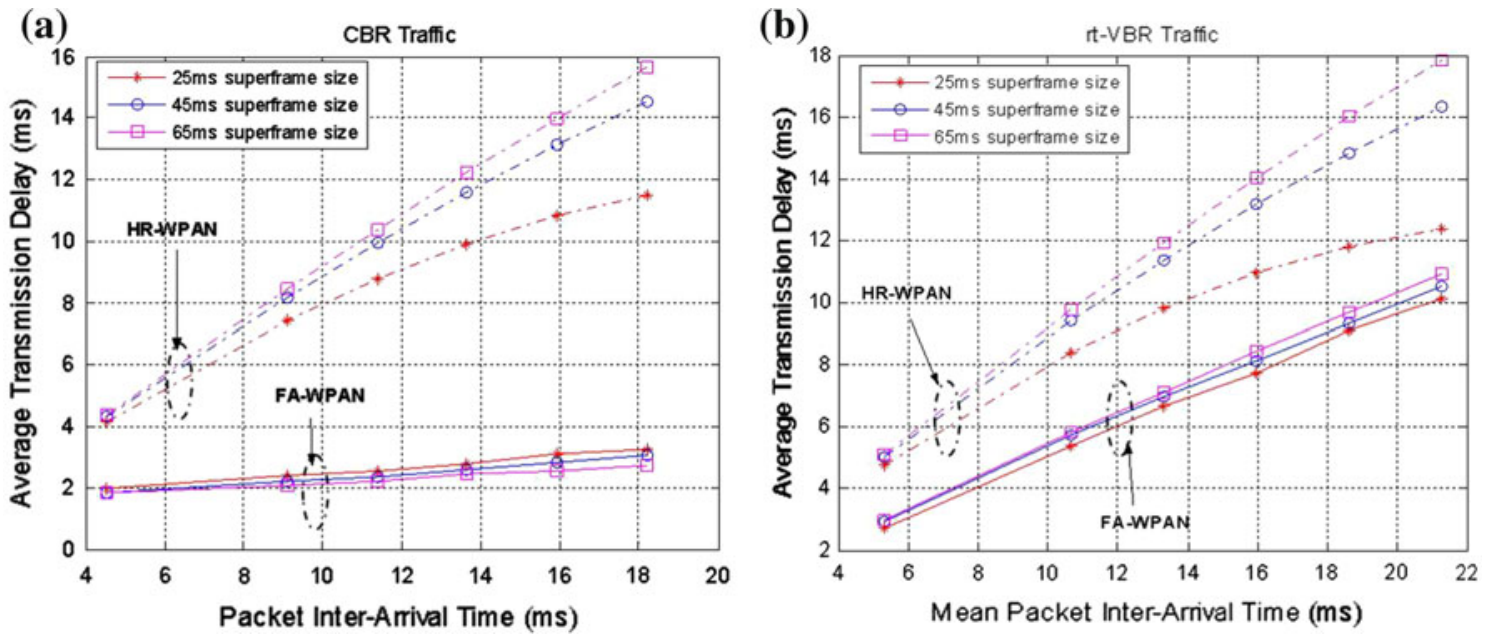

Fig. 9 Average transmission delay as a function of the packet inter arrival time for different superframe sizes

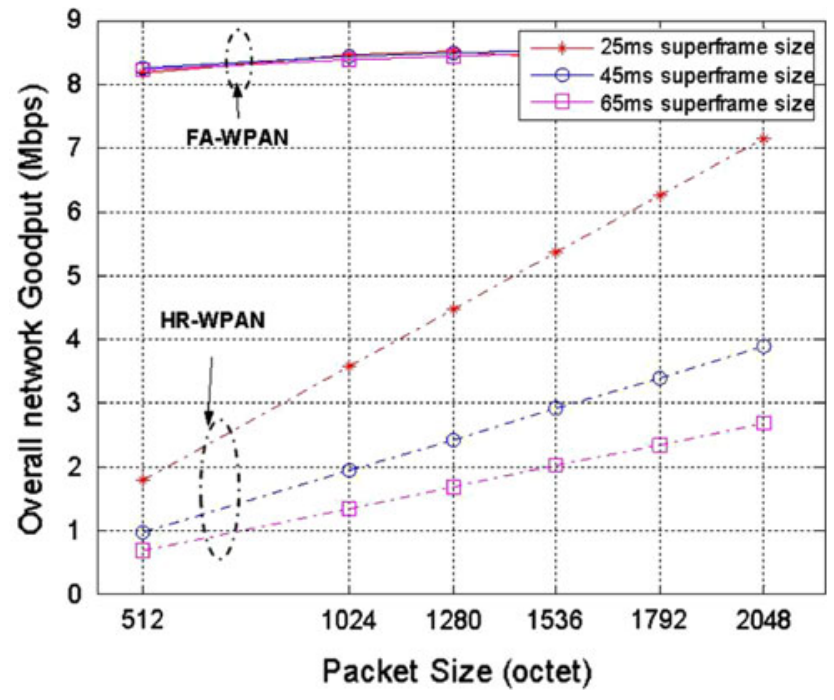

Fig. 10 Overall network goodput as a function of the packet size

bound for MPEG-4 traffic in [6-9], the higher delay bound multipliers than 1.5 may not be practical.

Figure 12 illustrates the JFRs of both configurations as the functions of the number of flows. The 2048 octets packet size is used for both traffic types. Delay bounds for CBR and rt-VBR traffic are three times longer than the packet inter-arrival times of both traffics because previous evaluation shows the best performance in that delay bound. For FA-WPAN, the JFRs of both traffics are constant at $0 \%$, and then slightly increase where there are 20 flows. In the heavy load case, CTA allocation may not be synchronized with the packet arrival time because of overlapped CTAs. For HR-WPAN, the allocated CTA durations are reduced with increasing the number of flows so that it is not adequate to transmit all pending packets. As Fig. 12 is shown, the JFR of proposed scheme also starts increasing from 20 flows. If the number of flows is large enough (more than 20), the proposed scheme cannot be able to meet the delay requirements. However, the notice obtained from the results is that the proposed scheme can reliably deal with higher number of multimedia traffic flows than the legacy HR-WPAN can.

Now, the effect of the rate adaptation is evaluated in Fig. 13. In this simulation, the superframe size is set to $35 \mathrm{~ms}$ and the packet size is 2048 octets. The proposed protocol is compared with two other protocols. The first one is the non-rate-adaptive protocol in which an initially chosen data rate is not changed until the flow is completed. The second one is a rate-adaptive protocol which adopts the transmission rate according to the network performance as described in IEEE 802.15.3 standard and Sect. 2.2. Under this protocol, whenever a receiver receives 10 packets, it sends the sender the received packet history such as the number of failed packets. If more than two out of the 10 packets are unsuccessfully transmitted, the sender reduces the next lower data rate. Otherwise, it increases the next higher data rate. In Fig 12, the first and second protocols are labeled as Non-Rate-Adaptation and IEEE 802.15.3, respectively. The time varying nature of the wireless channel is described by its Doppler spread and coherence time, which are inversely proportional to one another. In our simulation, we consider the effect of the change of the Doppler spread and coherence time with the Ricean parameter $0 \mathrm{~dB}$. Since WPAN is targeting at home or office environments, Doppler frequency varies from $1 \mathrm{~Hz}$ up to $8 \mathrm{~Hz}$ corresponding to the pedestrian speed of $1 \mathrm{~m} / \mathrm{s}$. From [21], the coherence time is obtained as

$T_{c}=\frac{1}{f_{m}}$

where $f_{m}$ is Doppler frequency. As shown in Fig. 13a, the proposed scheme has the lowest PER over the other two schemes. The results illustrate that the other two schemes 
(a)

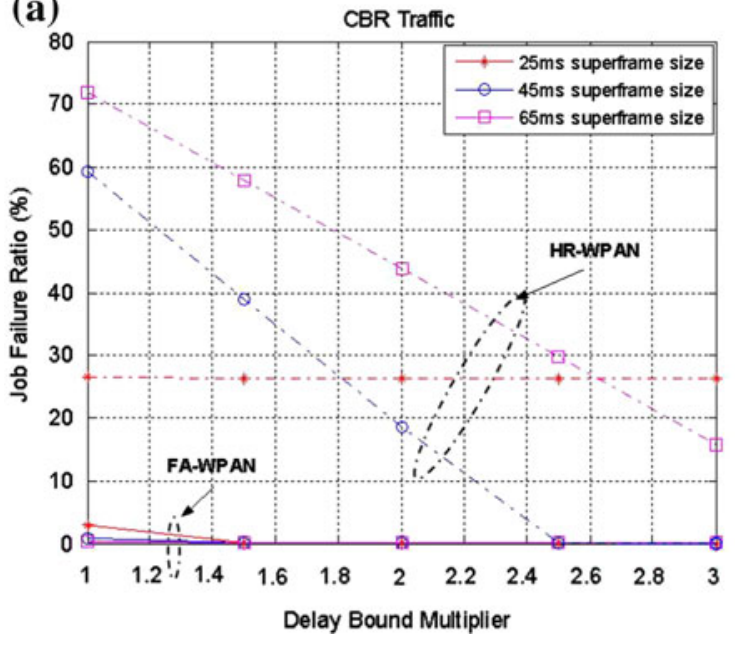

Fig. 11 Job failure ratio as a function of the delay bound multiplier

(a)

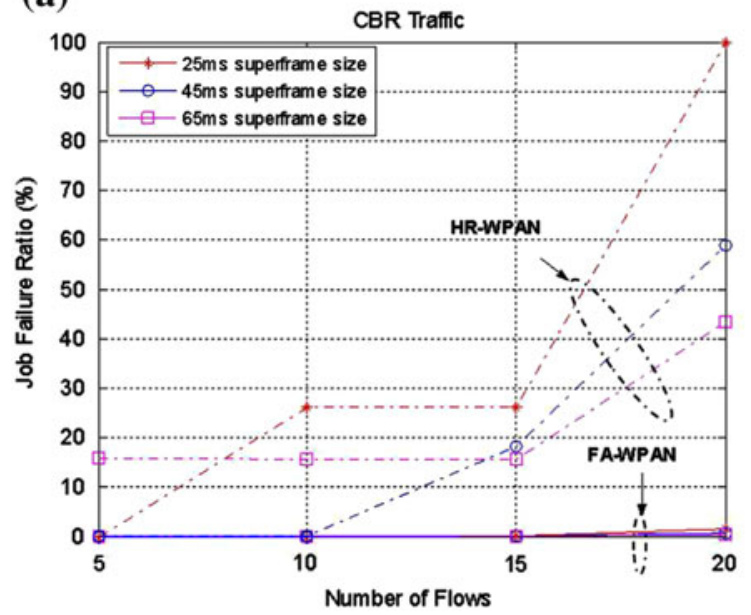

Fig. 12 Job failure ratio as a function of the number of flows

can hardly adapt to the varying wireless channel. Figure 13b shows the PER performance as a function of the Ricean parameter with $8 \mathrm{~Hz}$ Doppler frequency. The PERs for all schemes decrease with increasing Ricean parameter, which means improving channel condition. We observe that the PER of FA-WPAN is up to $78 \%$ less than that of Non-Rate-Adaptation and $76 \%$ less than that of IEEE 802.15.3.

\section{Conclusions}

In this paper, we propose a channel time allocation algorithm assisted by feedback information. The proposed scheme targets at delay-bounded applications in HRWPAN. The proposed algorithm initially allocates CTAs based on the statistical packet inter-arrival time, which is informed by each DEV using a modified CTRq command.

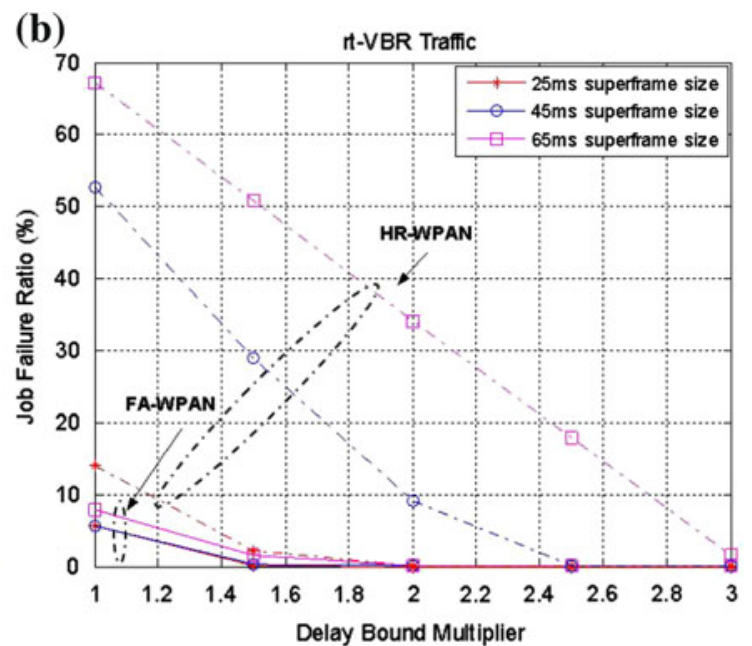

(b)

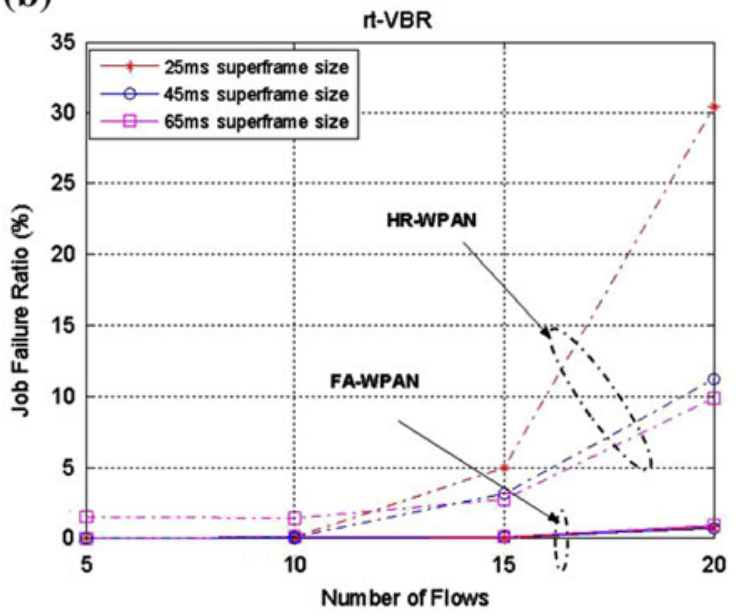

The initially allocated CTAs are dynamically relocated by utilizing feedback information in order to synchronize CTA to the packet arrival time and to allocate sufficient channel time for pending packets. We verify the performance enhancement by the extensive simulations. From the simulations, we have shown that the proposed scheme can achieve significant performance improvements over other comparative CTA allocation schemes. We note that the performance of the proposed scheme is not influenced by variable factors such as the superframe size, the delay bound, and the number of flows. The proposed method shows smaller JFR and shorter transmission delay than the HR-WPAN standard. Furthermore, a rate adaptation scheme is proposed to cope with the time varying wireless channel. In the scheme, the rate is selected according to the channel estimation in the physical layer. The proposed scheme reduces the packet error rate up to $89 \%$ of that of the adaptation scheme in the IEEE 802.15.3 
(a)

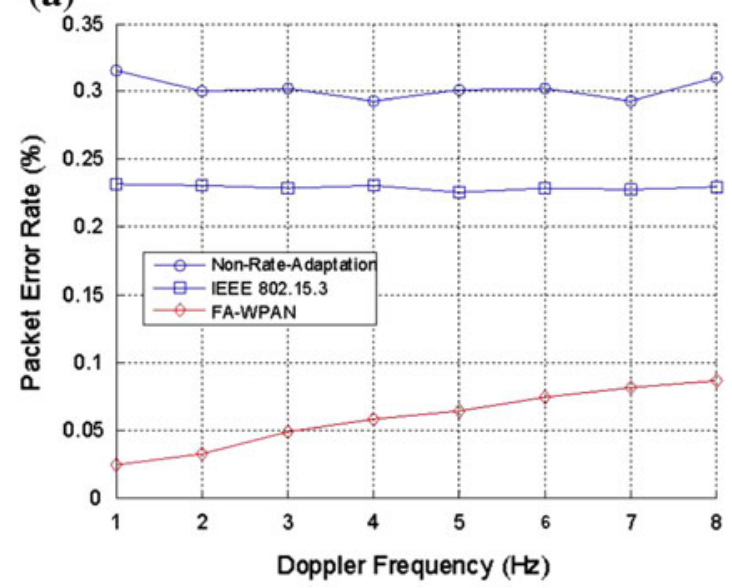

(b)

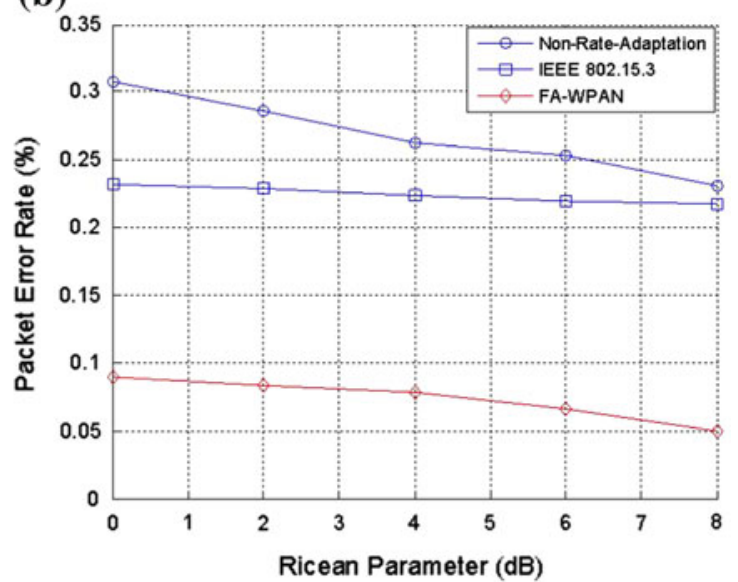

Fig. 13 Packet error rate comparisons as a function of a Doppler frequency and $\mathbf{b}$ ricean parameter

Acknowledgments This research was supported in part by the U.S. National Science Foundation under grant ANI-0220287, and by the MKE (The Ministry of Knowledge Economy), Korea, under the ITRC (Information Technology Research Center) support program supervised by the IITA (Institute for Information Technology Advancement) (IITA-2009-C1090-0902-0003).

\section{References}

1. Gandolfo, P. \& Allen, J. (2002). "802.15.3 Overview/Update," The WiMEDIA alliance.

2. Part 15.1: Wireless Medium Access Control (MAC) and Physical Layer (PHY). (2002). Specifications for Wireless Personal Area Networks (WPANs), IEEE Std. 802.15.1.

3. Part 15.3: Wireless Medium Access Control (MAC) and Physical Layer (PHY). (2003). Specifications for High Rate Wireless Personal Area Networks (WPANs), IEEE Std. 802.15.3.

4. Federal Communications Commission (FCC 02-48). (2002). "Revision of Part 15 regarding ultra-wideband transmission," ET Docket 98-153, First report and order.

5. Batra, A. (2003). "Multi-band OFDM physical layer proposal,". IEEE 802.15-03/267r6.

6. Mangharam, R. \& Demirhan, M. (2002). "Performance and simulation analysis of 802.15.3 QoS," IEEE 802.15-02/297r1.

7. Kang, C. G., Ahn, C. W., Jang, K. H., \& Kang, W. S. (2000). Contention-free distributed dynamic reservation MAC protocol with deterministic scheduling (C-FD3R MAC) for wireless ATM networks. IEEE Journal on Selected Areas in Communications, 18, 1623-1635. doi:10.1109/49.872951.

8. Torok, A., Vajda, L., Youn, K. -J., \& June S. -D. (2004). "Superframe formation algorithms in 802.15.3 networks,". IEEE WCNC'04 (pp. 1008-1013). Atlanta, Georgia.

9. Rhee, S. H., Chung, K., Kim, Y., Yoon, W., \& Chang, K. S. (2004)."An application-aware MAC scheme for IEEE 802.15.3 high-rate wpan,". In Proc. IEEE WCNC'04 (pp. 1018-1023). Atlanta, Georgia.

10. Moradi, S., \& Wong, V. W. S. (2007)."Technique to Improve MPEG-4 Traffic Schedulers in IEEE 802.15.3 WPANs,". In Proc. IEEE ICC'07. Glasgow, Scotland.

11. Brabenac, C. (2002). "MAC Performance enhancements for AltPHY," IEEE 802.15-02/472r0.
12. Kim, B. -S., Fang, Y., \& Wong, T. F. (2004)"Rate-adaptive MAC protocol in high rate personal area networks,". In Proc. IEEE WCNC'04 (pp. 1394-1399). Atlanta, Georgia.

13. Liu, K. -H., Rutagemwa, H., Shen, X., \& Mark, J. W. (2007). Efficiency and goodput analysis of Dly-ACK in IEEE 802.15.3. IEEE Transactions on Vehicular Technology, 56(6), 3888-3898. doi:10.1109/TVT.2007.904547.

14. Holland, G., Vaidya, N., \& Bahl, P. (2001)."A rate-adaptive MAC protocol for multi-hop wireless networks,". In Proc. ACM MOBICOM'01 (pp. 236-251).

15. IEEE 802.11 WG. (2004), Draft supplement to STANDARD FOR telecommunications and information exchange between systems-LAN/MAN Specific requirements-Part 11: Wireless Medium Access Control (MAC) and physical layer (PHY) specifications: Medium Access Control (MAC) Enhancements for Quality of Service (QoS), IEEE 802.11e/D8.0.

16. Grilo, A., Macedo M., \& Nunes, M. (2003). "A scheduling algorithm for QoS support in IEEE802.11E networks," IEEE wireless communications (pp. 36-43).

17. Fitzek, F. H. P., \& Reisslein, M. (2001). MPEG-4 and H.263 video traces for network performance evaluation. IEEE Network, (pp. 40-54). doi:10.1109/65.967596.

18. Kim, B. -S., Fang, Y., Wong, T. F., \& Kwon, Y. (2003)." Dynamic fragmentation scheme for rate-adaptive wireless LANs,". In Proc. IEEE PIMRC'03 (pp. 2591-2595).

19. DVD-Video Audio Coding. http://www.disctronics.co.uk/ technology/dvdvideo/dvdvid_audenc.htm.

20. MPEG-4 and H.263 Video Traces for Network Performance Evaluation (2002). http://www-tkn.ee.tu-berlin.de/research/ trace/trace.html.

21. Rappaport, T. S. (1996)."Wireless communications: principles and practices," (pp. 69-185). Prentice Hall.

22. K. Siwiak, \& Ellis, J. (2002). "SG3a Alternate PHY selection criteria," IEEE P802.15-02/105r20.

23. Punnoose, R. J., Nikitin, P. V., \& Stancil, D. D. (2000)."Efficient simulation of ricean fading within a packet simulator,". In Proc. IEEE VTC'OO (pp. 764-767).

24. Proakis, J. G. (1995) "Digital communications" 3rd ed. New York:McGraw-Hill (pp. 257-282).

25. Lo, S. -C., Lee, G., \& Chen, W. -T. (2003). An Efficient Multipolling Mechanism for IEEE 802.11 Wireless LANs. IEEE Transactions on Computers, 76, 4-778.

26. Koutsakis, P., Psychis, S., \& Paterakis, M. (2001).”On the integration of MPEG-4 video stream with voice and e-mail data 
packet traffic over wireless picocellular networks,". In Proc. IEEE PIMRC'01 (pp. 60-64).

\section{Author Biographies}

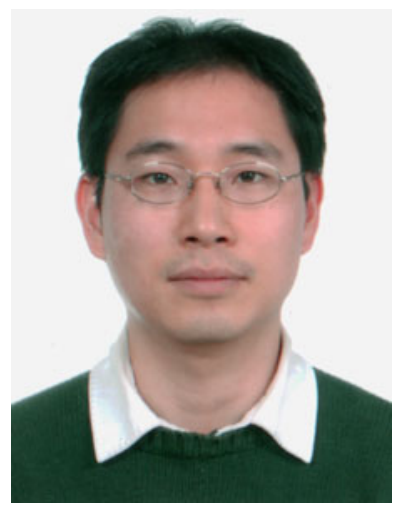

Byung-Seo Kim received his B.S. degree in Electrical Engineering from In-Ha University, In-Chon, Korea in 1998 and his M.S. and Ph.D. degrees in Electrical and Computer Engineering from the University of Florida in 2001 and 2004, respectively. Between 1997 and 1999, he worked for Motorola Korea Ltd., PaJu, Korea as a Computer Integrated Manufacturing (CIM) Engineer in Advanced Technology Research \& Development (ATR\&D). From January 2005 to August 2007, he worked for Motorola Inc., Schaumburg Illinois, as a Senior Software Engineer in Networks and Enterprises. Since September 2007, he has been an Assistant Professor at the Department of Computer and Information Communication Engineering in HongIk University, Korea. His research interests include the design and development of efficient link-adaptable MAC protocols, cross layer architectures, Multi-MAC structures and resource allocation algorithms for wireless networks. He also worked in physical layer design for PLC and $802.11 \mathrm{n}$. He is currently an IEEE member.

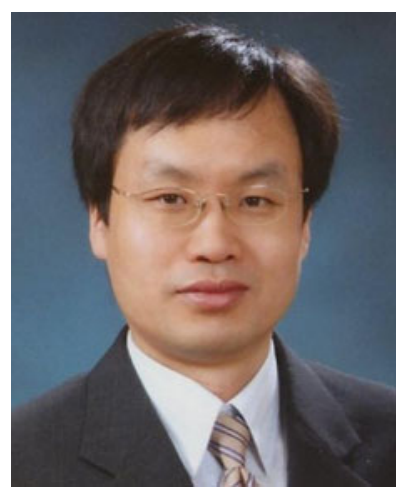

Sung Won Kim received his B.S. and M.S. degrees from the Department of Control and Instrumentation Engineering, Seoul National University, Korea, in 1990 and 1992, respectively, and his Ph.D. degree from the School of Electrical Engineering and Computer Sciences, Seoul National University, Korea, in August 2002. From January 1992 to August 2001, he was a Researcher at the Research and Development Center of LG Electronics, Korea. From August 2001 to August 2003, he was a Researcher at the Research and Development Center of AL Tech, Korea. From August 2003 to February 2005, he was a Postdoctoral Researcher in the Department of Electrical and Computer Engineering, University of Florida, Gainesville, USA. In March 2005, he joined the School of Electrical Engineering and Computer Science, Yeungnam University, Gyeongsangbuk-do, Korea, where he is currently an Associate Professor. His research interests include resource management, wireless networks, mobile networks, performance evaluation, and embedded systems.

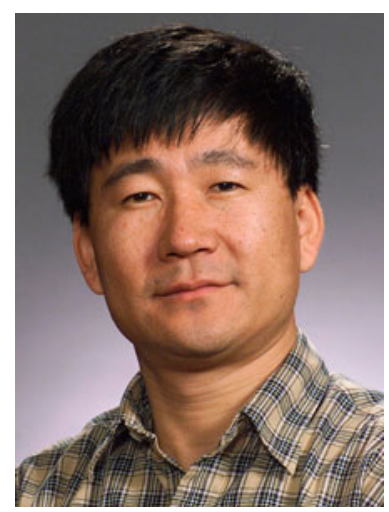

Yuguang Fang received a Ph.D. degree in Systems Engineering from Case Western Reserve University in January 1994 and a Ph.D. degree in Electrical Engineering from Boston University in May 1997. He was an Assistant Professor in the Department of Electrical and Computer Engineering at New Jersey Institute of Technology from July 1998 to May 2000. He then joined the Department of Electrical and Computer Engineering at University of Florida in May 2000 as an Assistant Professor, got an early promotion to an Associate Professor with tenure in August 2003 and a Professor in August 2005. He has published over 150 papers in refereed professional journals and conferences. He received the National Science Foundation Faculty Early Career Award in 2001 and the Office of Naval Research Young Investigator Award in 2002. He has served on many editorial boards of technical journals including IEEE Transactions on Communications, IEEE Transactions on Wireless Communications, IEEE Transactions on Mobile Computing and ACM Wireless Networks. He is a fellow of the IEEE.

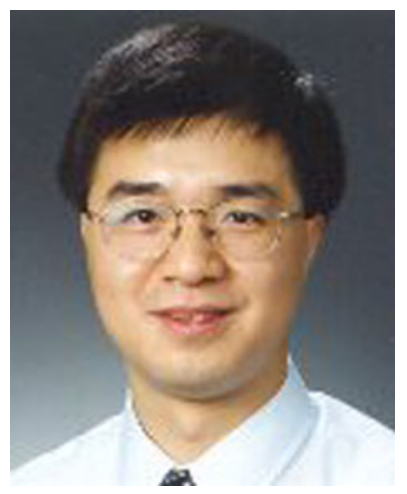

Tan F. Wong received the B.Sc. degree (1st class honors) in Electronic Engineering from the Chinese University of Hong Kong in 1991, and the M.S.E.E. and Ph.D. degrees in Electrical Engineering from Purdue University in 1992 and 1997, respectively. He was a Research Engineer working on the high speed wireless networks project in the Department of Electronics at Macquarie University, Sydney, Australia. He also served as a Post-Doctoral Research Associate in the School of Electrical and Computer Engineering at Purdue University. Since August 1998 he has been with the University of Florida, where he is currently an Associate Professor of Electrical and Computer Engineering. He was Editor for Wideband and Multiple Access Wireless Systems for the IEEE Transactions On Communications and was the Editor for the IEEE Transactions On Vehicular Technology from 2003 to 2006. 